Konstanz University • Theory Group

〈Gravity | Quantum Theory | Optics 〉

Preprint KONS-RGKU-95-07

\title{
Generalized Unruh effect and Lamb shift for atoms on arbitrary stationary trajectories
}

\author{
Jürgen Audretsch円, Rainer Müller|2 and Markus Holzmann \\ Fakultät für Physik der Universität Konstanz \\ Postfach 5560 M 674, D-78434 Konstanz, Germany
}

\begin{abstract}
We study the spontaneous de-excitation and excitation of accelerated atoms on arbitrary stationary trajectories ("generalized Unruh effect"). We consider the effects of vacuum fluctuations and radiation reaction separately. We show that radiation reaction is generally not altered by stationary acceleration, whereas the contribution of vacuum fluctuations differs for all stationary accelerated trajectories from its inertial value. Spontaneous excitation from the ground state occurs for all accelerated stationary trajectories and is therefore the "normal case". We furthermore show that the radiative energy shift ("Lamb shift") of a two-level atom is modified by acceleration for all stationary trajectories. Again only vacuum fluctuations give rise to the shift. Our results are illustrated for the special case of an atom in circular motion, which may be experimentally relevant.

PACS numbers: $32.80-\mathrm{t} ;$ 42.50-p.
\end{abstract}

\section{Introduction}

Recently, a new physical picture for the spontaneous excitation of a uniformly accelerated two-level atom (the Unruh effect [1]) has been put forward [2]. Following a quantum optical approach it is based on the distinction of the two competing mechanisms which operate to excite an atom in the quantum vacuum: vacuum fluctuations and radiation reaction. Vacuum fluctuations tend to excite an atom in its ground state and de-excite it in an excited state. On the other hand, radiation reaction leads always to a loss of internal excitation energy. For an inertial atom in the ground state, the two contributions cancel exactly, so that a very sublime balance between vacuum fluctuations and radiation reaction prevents the spontaneous excitation of the atom [3]. If the atom is in the excited state, both contribution add up to the well-known spontaneous emission rate. It has been shown in [2] (see also [4]) that uniform acceleration will disturb this balance. Spontaneous transitions from the ground state to the excited state become possible: the Unruh effect. Similarly, the rate of spontaneous emission is modified from its inertial value for a uniformly accelerated atom.

\footnotetext{
${ }^{1}$ e-mail: Juergen.Audretsch@uni-konstanz.de

${ }^{2}$ Present address: Sektion Physik der Universität München, Theresienstr. 37, D-80333 München, Germany

e-mail: Rainer.Mueller@physik.uni-muenchen.de
} 
It is interesting to investigate the "fine structure" of these processes by considering vacuum fluctuations and radiation reaction separately. In the case of uniform acceleration, the contribution of vacuum fluctuations is changed from its inertial value, while the contribution of radiation reaction remains exactly the same as for the inertial atom. Their combined action leads to the spontaneous excitation or de-excitation described above.

In this paper, we will concentrate on more general states of motion of the atom. We consider arbitrary stationary trajectories in Minkowski space, which can be characterized as a motion along a timelike Killing vector field or, equivalently, by the condition that the geodesic distance between two points on the trajectory depends only on their proper time difference [5]. We will ask for which of these trajectories there is a spontaneous excitation of the atom ("generalized Unruh effect"). Furthermore we will discuss whether it is a general trait that the contribution of radiation reaction is not modified by the acceleration or whether this holds only in the special case of uniform acceleration. Another question is whether it is true also for more general trajectories that the atom is excited because it perceives the vacuum fluctuations differently.

Our results are summarized in a number of theorems. The first one shows that the contribution of radiation reaction is the same for all stationary accelerated trajectories as in the inertial case. Accordingly, radiation reaction is a purely local concept that is not sensitive to the actual state of motion of the atom. This can be understood heuristically: the field is radiated away by the atom with the velocity of light. Since the atom's trajectory is timelike, the only moment it can act back on the atom is precisely at the time it is emitted. The mechanism of radiation reaction is thus restricted to act at this point of the trajectory alone.

Next we show that the contribution of vacuum fluctuations differs for all atoms on accelerated stationary trajectories from that of an inertial atom. Together, these two results demonstrate that a generalized Unruh effect takes place for all stationary trajectories except the inertial ones. In this sense, the spontaneous excitation of an atom is the normal case and the non-occurrence of the Unruh effect for inertial atoms is an exception.

The second main part of the paper is concerned with the radiative energy shift of accelerated atoms. The "Lamb shift" for a uniformly accelerated two-level atom has been calculated already in [6]. This shift can be explained qualitatively as follows: For a uniformly accelerated atom, the Minkowski vacuum appears as a thermal bath of particles called Rindler quanta. The AC Stark shift associated with these particles is responsible for the atom's modified Lamb shift.

In Sec. 4, we will investigate the Lamb shift for atoms on arbitrary stationary trajectories, where a simple interpretation in terms of Rindler particles is no longer possible. Already for an inertial atom, the energy shift diverges and must be regularized, for example by imposing a cutoff energy. Consequently, it may be difficult to isolate the modifications introduced by the acceleration. We overcome this problem by referring to a recently proved theorem [7] that connects the radiative energy shifts to the transition rates between the levels, which are much easier to calculate. In this way, we can show that the accelerationinduced modification of the Lamb shift is always finite. This allows an unambiguous separation into a (divergent) inertial part and a finite correction caused by the acceleration.

We will then consider the individual contributions to the Lamb shift. We will show that the effect of radiation reaction is the same for an atom on an arbitrary stationary trajectory as for an inertial one. It is equal for both levels and does not contribute to the relative shift. Contrarily, the effect of vacuum fluctuations is modified for any accelerated stationary trajectory. The total shift differs always from its inertial value.

As an illustration of our results, we treat in Sec. 5 an atom in circular motion. The spontaneous excitation for a two-level atom on such a trajectory has been treated previously [8, 9, 10] and may be of experimental relevance. We discuss the contributions of vacuum fluctuations and radiation reaction separately and determine the evolution of the mean atomic excitation energy towards its equilibrium value. Furthermore, we identify the Einstein coefficients for spontaneous excitation and de-excitation. Finally, the Lamb shift for a circulating atom is calculated by applying the theorem of Ref. [7]. The correction due to the acceleration is isolated and compared with the uniformly accelerated case. 


\section{Vacuum fluctuations and radiation reaction: the general for- malism}

As a basis for the discussion of the behaviour of accelerated atoms in arbitrary stationary motion in Special Relativity, we will briefly recall the model and the Heisenberg picture formalism that was used to treat the case of uniformly accelerated atoms in previous publications [2, 6. We consider a pointlike twolevel atom on an arbitrary stationary space-time trajectory $x(\tau)$ which interacts with a scalar quantum field $\phi . \tau$ denotes the proper time on the trajectory. A characterization of stationary motion in Minkowski space has been given by Letaw [5], resulting in two equivalent conditions: (a) the trajectory follows the orbits of a timelike Killing vector field, or (b) the geodesic distance between two points $x(\tau)$ and $x\left(\tau^{\prime}\right)$ on the trajectory depends only on the proper time interval $\tau-\tau^{\prime}$.

Property (a), mentioned above, ensures that the metric along the path of the atom does not change and guarantees the existence of stationary states. For our two-level atom, these will be called $|+\rangle$ and $|-\rangle$, with energies $\pm \frac{1}{2} \omega_{0}$. The atom's undisturbed Hamiltonian with respect to its proper time $\tau$ can then be written

$$
H_{A}(\tau)=\omega_{0} R_{3}(\tau)
$$

where $R_{3}=\frac{1}{2}|+\rangle\left\langle+\left|-\frac{1}{2}\right|-\right\rangle\langle-|$ is the pseudospin operator commonly used in the description of two-level atoms [11, 12].

The free Hamiltonian of the scalar quantum field that governs its time evolution with respect to $\tau$ is

$$
H_{F}(\tau)=\int d^{3} k \omega_{\vec{k}} a_{\vec{k}}^{\dagger} a_{\vec{k}} \frac{d t}{d \tau}
$$

with creation and annihilation operators $a_{\vec{k}}^{\dagger}, a_{\vec{k}}$. Atom and field are coupled by the interaction

$$
H_{I}(\tau)=\mu R_{2}(\tau) \phi(x(\tau))
$$

where $\mu$ is a small coupling constant, $R_{2}=\frac{1}{2} i\left(R_{-}-R_{+}\right)$, and $R_{+}=|+\rangle\left\langle-\left|, R_{-}=\right|-\right\rangle\langle+|$. Note that the atom-field coupling (3) is effective only on the trajectory of the atom.

We can now write down the Heisenberg equations of motion for the atom and field observables. The field is always considered to be in its special relativistic vacuum state $|0\rangle$ ( Minkowski vacuum). To isolate the contributions of vacuum fluctuations and radiation reaction to the rate of change of atomic observables, their solution for the field $\phi$ is split into its free or vacuum part $\phi^{f}$, which is present even in the absence of the coupling, and its source part $\phi^{s}$, which represents the field generated by the atom itself. If we consider now the effects of $\phi^{f}$ and $\phi^{s}$ separately in the Heisenberg equations of an arbitrary atomic observable $G$, we obtain the individual contributions of vacuum fluctuations and radiation reaction to the rate of change of $G$. Following Dalibard, Dupont-Roc and cohen-Tannoudji [3], we choose a symmetric ordering between atom and field variables. Below we repeat only the necessary definitions and central expressions of [2] and [6].

Our first aim is the discussion of spontaneous emission and spontaneous excitation from the ground state $|-\rangle$ which we will call generalized Unruh effect. We therefore concentrate on the mean atomic excitation energy $\left\langle H_{A}(\tau)\right\rangle$. It has been shown in [2] that the contributions of vacuum fluctuations (vf) and radiation reaction (rr) to the rate of change of $\left\langle H_{A}\right\rangle$ can be written (cf. [3, 13])

$$
\begin{aligned}
& \left\langle\frac{d H_{A}(\tau)}{d \tau}\right\rangle_{v f}=2 i \mu^{2} \int_{\tau_{0}}^{\tau} d \tau^{\prime} C^{F}\left(x(\tau), x\left(\tau^{\prime}\right)\right) \frac{d}{d \tau} \chi^{A}\left(\tau, \tau^{\prime}\right), \\
& \left\langle\frac{d H_{A}(\tau)}{d \tau}\right\rangle_{r r}=2 i \mu^{2} \int_{\tau_{0}}^{\tau} d \tau^{\prime} \chi^{F}\left(x(\tau), x\left(\tau^{\prime}\right)\right) \frac{d}{d \tau} C^{A}\left(\tau, \tau^{\prime}\right) .
\end{aligned}
$$

with |\rangle$=|a, 0\rangle$ representing the atom in the state $|a\rangle$ and the field in the Minkowski vacuum state $|0\rangle$.They are expressed in terms of the statistical functions of the atom

$$
\begin{aligned}
C^{A}\left(\tau, \tau^{\prime}\right) & :=\frac{1}{2}\left\langle a\left|\left\{R_{2}^{f}(\tau), R_{2}^{f}\left(\tau^{\prime}\right)\right\}\right| a\right\rangle \\
\chi^{A}\left(\tau, \tau^{\prime}\right) & :=\frac{1}{2}\left\langle a\left|\left[R_{2}^{f}(\tau), R_{2}^{f}\left(\tau^{\prime}\right)\right]\right| a\right\rangle .
\end{aligned}
$$


and of the field

$$
\begin{aligned}
C^{F}\left(x(\tau), x\left(\tau^{\prime}\right)\right) & :=\frac{1}{2}\left\langle 0\left|\left\{\phi^{f}(x(\tau)), \phi^{f}\left(x\left(\tau^{\prime}\right)\right)\right\}\right| 0\right\rangle, \\
\chi^{F}\left(x(\tau), x\left(\tau^{\prime}\right)\right) & :=\frac{1}{2}\left\langle 0\left|\left[\phi^{f}(x(\tau)), \phi^{f}\left(x\left(\tau^{\prime}\right)\right)\right]\right| 0\right\rangle .
\end{aligned}
$$

$C^{A}$ and $C^{F}$ are called symmetric correlation functions, $\chi^{A}$ and $\chi^{F}$ linear susceptibilities. Note that the dependence on the trajectory $x(\tau)$ of the atom in (4) and (5) is contained entirely in the statistical functions of the field, which have to be evaluated at two points on the atom's world line. The atom registers the influence of the Minkowski vacuum when following its specific space-time trajectory.

The explicit form of the statistical functions of the atom is given by

$$
\begin{aligned}
C^{A}\left(\tau, \tau^{\prime}\right) & =\frac{1}{2} \sum_{b}\left|\left\langle a\left|R_{2}^{f}(0)\right| b\right\rangle\right|^{2}\left(e^{i \omega_{a b}\left(\tau-\tau^{\prime}\right)}+e^{-i \omega_{a b}\left(\tau-\tau^{\prime}\right)}\right), \\
\chi^{A}\left(\tau, \tau^{\prime}\right) & =\frac{1}{2} \sum_{b}\left|\left\langle a\left|R_{2}^{f}(0)\right| b\right\rangle\right|^{2}\left(e^{i \omega_{a b}\left(\tau-\tau^{\prime}\right)}-e^{-i \omega_{a b}\left(\tau-\tau^{\prime}\right)}\right),
\end{aligned}
$$

where $\omega_{a b}=\omega_{a}-\omega_{b}$ and the sum extends over a complete set of atomic states. The statistical functions of the field are

$$
\begin{aligned}
C^{F}\left(x(\tau), x\left(\tau^{\prime}\right)\right) & =\frac{1}{8 \pi^{2}} \frac{1}{|\Delta \vec{x}|}\left(\frac{\mathcal{P}}{\Delta t+|\Delta \vec{x}|}-\frac{\mathcal{P}}{\Delta t-|\Delta \vec{x}|}\right) \\
& =-\frac{1}{8 \pi^{2}}\left(\frac{1}{(\Delta t+i \epsilon)^{2}-|\Delta \vec{x}|^{2}}+\frac{1}{(\Delta t-i \epsilon)^{2}-|\Delta \vec{x}|^{2}}\right), \\
\chi^{F}\left(x(\tau), x\left(\tau^{\prime}\right)\right) & =\frac{i}{8 \pi} \frac{1}{|\Delta \vec{x}|}(\delta(\Delta t+\Delta|\vec{x}|)-\delta(\Delta t-|\Delta \vec{x}|)), \\
& =\frac{1}{8 \pi^{2}}\left(\frac{1}{(\Delta t+i \epsilon)^{2}-|\Delta \vec{x}|^{2}}-\frac{1}{(\Delta t-i \epsilon)^{2}-|\Delta \vec{x}|^{2}}\right),
\end{aligned}
$$

where $\Delta t=t(\tau)-t\left(\tau^{\prime}\right), \Delta \vec{x}=\vec{x}(\tau)-\vec{x}\left(\tau^{\prime}\right)$ and $\mathcal{P}$ denotes the principal value.

In a next step, we consider the "Lamb shift" of the two-level atom, i. e. the radiative energy shifts of its levels due to the coupling to the quantum vacuum. To this end, one studies the evolution of an atomic observable $G$ and traces out the field degrees of freedom in its equations of motion. It is then possible to identify an effective Hamiltonian for the atom which acts in addition to $H_{A}$ (cf. [3, 13]). The expectation value of $H_{\text {eff }}$ in an atomic state $|a\rangle$ gives the radiative shift of this level. Again the division of the field into free and source part allows the identification of the contributions of vacuum fluctuations and radiation reaction. As has been shown in [6], the radiative energy shifts of level $|a\rangle$ are given by

$$
\begin{aligned}
& \left(\delta E_{a}\right)_{v f}=-i \mu^{2} \int_{\tau_{0}}^{\tau} d \tau^{\prime} C^{F}\left(x(\tau), x\left(\tau^{\prime}\right)\right) \chi^{A}\left(\tau, \tau^{\prime}\right), \\
& \left(\delta E_{a}\right)_{r r}=-i \mu^{2} \int_{\tau_{0}}^{\tau} d \tau^{\prime} \chi^{F}\left(x(\tau), x\left(\tau^{\prime}\right)\right) C^{A}\left(\tau, \tau^{\prime}\right) .
\end{aligned}
$$

As we restrict ourselves to the model of a two level atom the statistical functions of the atom (10) and (11) can be simplified: The level spacing $\omega_{a b}$ is given by $\left|\omega_{a b}\right|=\omega_{o} \delta_{a, b}$ and the matrix element $\left|\left\langle a\left|R_{2}^{f}(0)\right| b\right\rangle\right|^{2}$ is reduced to

$$
\left|\left\langle a\left|R_{2}^{f}(0)\right| b\right\rangle\right|^{2}=\frac{1}{4}\left(1-\delta_{a, b}\right) .
$$

Hence the summation in (10) and (11) breaks down. In the following theorems we nevertheless use the unevaluated formulas (10) and (11) to stress the structure of the results so that possible extensions to multi-level atoms are easily made (cf. [7]). 


\section{Relaxation rates for atoms on arbitrary stationary trajecto- ries}

Based on the formalism presented above, we will discuss in this section some general features of the relaxation rates (4) and (5) for atoms in arbitrary stationary motion. Two special cases, discussed in a previous paper [2], are an unaccelerated atom in uniform motion (or at rest) and a uniformly accelerated atom with constant acceleration $a$. It was found in 20 that for a uniformly accelerated atom the contribution of radiation reaction (42) to $\left\langle d H_{A} / d \tau\right\rangle$ is the same as for an atom at rest. On the other hand, the contribution of vacuum fluctuations is changed by the influence of acceleration. This means that spontaneous excitation of a uniformly accelerated atom in the ground state becomes possible and provides a physical picture of the Unruh effect.

The question arises: is this structure a special feature of the uniformly accelerated trajectory or does it appear in a similar way for other stationary trajectories too? We will prove two theorems which show that indeed spontaneous excitation of the atom takes place for any non-inertial trajectory

Theorem 1: The contribution of radiation reaction to the rate of change $\left\langle\frac{d H_{A}}{d \tau}\right\rangle$ of the mean atomic energy is for all stationary trajectories equal to that of an atom at rest:

$$
\left\langle\frac{d H_{A}(\tau)}{d \tau}\right\rangle_{r r}=\sum_{b} \Gamma_{a b}^{r r}\left(\omega_{a b}\right)
$$

where

$$
\Gamma_{a b}^{r r}(\omega):=-\frac{\mu^{2}}{4 \pi} \omega^{2}\left|\left\langle a\left|R_{2}^{f}(0)\right| b\right\rangle\right|^{2} .
$$

Proof: In Eq. (5), the only dependence on the trajectory of the atom is through the linear susceptibility of the field. It is therefore sufficient to analyze $\chi^{F}$. We will show that it is equal in the distributional sense (i. e. integrated over an arbitrary test function) for all timelike trajectories. Starting from Eq. (13), we apply $\chi^{F}$ to a test function $f\left(\tau^{\prime}\right)$. Since its Fourier transform $\tilde{f}(\omega)$ exists, it can be split in a part $f^{(+)}\left(\tau^{\prime}\right)$ that vanishes for $\tau^{\prime} \rightarrow+i \infty$ and a part $f^{(-)}\left(\tau^{\prime}\right)$ that vanishes for $\tau^{\prime} \rightarrow-i \infty$ :

$$
f\left(\tau^{\prime}\right)=f^{(+)}\left(\tau^{\prime}\right)+f^{(-)}\left(\tau^{\prime}\right):=\int_{0}^{\infty} d \omega\left(\tilde{f}(\omega) e^{i \omega \tau^{\prime}}+\tilde{f}(-\omega) e^{-i \omega \tau^{\prime}}\right)
$$

We first treat the $f^{(+)}$part of the integral:

$$
\int d \tau^{\prime} f^{(+)}\left(\tau^{\prime}\right) \chi^{F}\left(x(\tau), x\left(\tau^{\prime}\right)\right)=\frac{1}{8 \pi^{2}} \int d \tau^{\prime} f^{(+)}\left(\tau^{\prime}\right)\left(\frac{1}{(\Delta t+i \epsilon)^{2}-|\Delta \vec{x}|^{2}}-\frac{1}{(\Delta t-i \epsilon)^{2}-|\Delta \vec{x}|^{2}}\right)
$$

We calculate the integral via the residue theorem and treat first the case where the contour can be closed in the upper half complex $\tau^{\prime}$ plane. Due to the $i \epsilon$ structure of the two parts of $\chi^{F}$, only the poles on the real $\tau^{\prime}$ axis contribute. We notice that for real $\tau^{\prime}$, the denominator of (18) vanishes only for lightlike separated points $x(\tau)$ and $x\left(\tau^{\prime}\right)$. Because our trajectory is timelike, the only contribution comes from $\tau^{\prime}=\tau$. We can therefore expand the denominator in (18) around this point:

$$
(\Delta t \pm i \epsilon)^{2}-|\Delta \vec{x}|^{2} \approx\left(\tau-\tau^{\prime}\right)^{2}\left[\left(\frac{d t\left(\tau^{\prime}\right)}{d \tau^{\prime}}\right)^{2}-\left(\frac{d \vec{x}\left(\tau^{\prime}\right)}{d \tau^{\prime}}\right)^{2}\right]_{\tau^{\prime}=\tau} \pm 2 i \epsilon\left(\frac{d t\left(\tau^{\prime}\right)}{d \tau^{\prime}}\right)_{\tau^{\prime}=\tau} .
$$

The term in square brackets is just the square of the four-velocity $u^{\mu} u_{\mu}=1$ and we obtain

$$
(\Delta t \pm i \epsilon)^{2}-|\Delta \vec{x}|^{2} \approx\left(\tau-\tau^{\prime} \pm i \epsilon\right)^{2}
$$

where we have absorbed the positive quantity $d t / d \tau^{\prime}$ in $\epsilon$.

The evaluation of the integral (18) is now straightforward. One finds

$$
\int d \tau^{\prime} f^{(+)}\left(\tau^{\prime}\right) \chi^{F}\left(x(\tau), x\left(\tau^{\prime}\right)\right)=\frac{i}{4 \pi} \int d \tau^{\prime} f^{(+)}\left(\tau^{\prime}\right) \delta^{\prime}\left(\tau-\tau^{\prime}\right) .
$$

For the $f^{(-)}$part which vanishes for $\tau^{\prime} \rightarrow-i \infty$ the contour must be closed in the lower half plane and the same result is found. By adding the two contributions, the desired result is obtained. 
Eq. (21) shows that for any accelerated trajectory, $\chi^{F}$ has the same functional form as for an inertial one. Note that it was not necessary to assume stationarity in deriving (21).

To complete the proof, we calculate from (5) and (10) the contribution of radiation reaction to the rate of change of $\left\langle H_{A}\right\rangle$ :

$$
\left\langle\frac{d H_{A}(\tau)}{d \tau}\right\rangle_{r r}=-2 i \mu^{2} \sum_{b} \omega_{a b}\left|\left\langle a\left|R_{2}^{f}(0)\right| b\right\rangle\right|^{2} \int_{0}^{+\infty} d u \chi^{F}(u) \sin \omega_{a b} u
$$

where $u=\tau-\tau^{\prime}$. The integrand is symmetric so that we can extend the lower limit to $-\infty$. The evaluation of the integral is trivial and we obtain (16) with (17). This expression is independent of the trajectory $x(\tau)$. It is the same formula we found already in $[2]$ for an inertial atom and a uniformly accelerated atom.

It is worth noting, that the structure of $(16)$ and (17) is maintained for multi-level atoms, since we did not use any properties restricted to the two-level model.

Theorem 2: The contribution of vacuum fluctuations to $\left\langle d H_{A} / d \tau\right\rangle$ differs for any accelerated stationary trajectory from that of an atom at rest.

Proof: Using (11), we write Eq. (4) in the form

$$
\left\langle\frac{d H_{A}(\tau)}{d \tau}\right\rangle_{v f}=\sum_{b} \Gamma_{a b}^{v f}\left(\omega_{a b}\right)
$$

with

$$
\Gamma_{a b}^{v f}(\omega):=-2 \mu^{2} \omega\left|\left\langle a\left|R_{2}^{f}(0)\right| b\right\rangle\right|^{2} \int_{0}^{\infty} d u C^{F}(u) \cos \omega u .
$$

We recognize that the integral appearing in this expression is the Fourier cosine transform of the symmetric correlation function $C^{F}(u)$. Hence, to show that (23) deviates from its inertial value for a noninertial form of $C^{F}(u)$, we can use the uniqueness theorem of the Fourier transformation: if $C^{F}(u)$ is of bounded variation and the integral converges, the Fourier integral is unique. The first requirement is trivially fulfilled for the monotonic function $C^{F}(u)$. To show that the second one is also met, we use the explicit form (12) of the symmetric correlation function. Apart from the convergence factor, the term appearing in the denominator is the geodesic distance $\sigma\left(\tau-\tau^{\prime}\right)$ between the two points $x(\tau)$ and $x\left(\tau^{\prime}\right)$ on the trajectory of the atom, expressed as a function of the proper time $\tau$. Now we use the fact that for any two points, the four-distance along their connecting geodesic is maximal. Therefore

$$
\sigma\left(\tau, \tau^{\prime}\right)>\left|\tau-\tau^{\prime}\right|
$$

Here

$$
\tau-\tau^{\prime}=\int_{\tau^{\prime}}^{\tau} d \tilde{\tau}
$$

is the four-distance as measured along the world line. Hence we always have

$$
\frac{1}{\sigma^{2}\left(\tau, \tau^{\prime}\right)}<\frac{1}{\left(\tau-\tau^{\prime}\right)^{2}}
$$

and the Fourier integral exists.

But the right-hand side of (27) is just the expression appearing in the symmetric correlation function for an inertial atom (cf. Eq. (35) of [2]). Thus we have

$$
C^{F}\left(x(\tau), x\left(\tau^{\prime}\right)\right)<C_{\text {inert }}^{F}\left(x(\tau), x\left(\tau^{\prime}\right)\right)
$$

for any two $\tau, \tau^{\prime}$. From the uniqueness of the Fourier transform we now can say that

$$
\Gamma_{a b}(\omega) \neq \Gamma_{a b}^{i n e r t}(\omega)
$$

for any accelerated stationary atom. Using (23), the proof for a two level atom is complete.

Consequence: Taking the contents of Theorems 1 and 2 together, we see that for an accelerated atom in its ground state, the balance between vacuum fluctuations and radiation reaction, which prevents spontaneous excitations for inertial atoms, is never maintained in the non-inertial case. Spontaneous transitions from the ground state to the excited state therefore take place on an arbitrary accelerated stationary trajectory, leading to the generalized Unruh effect. In addition, the spontaneous emission rate for an atom in the excited state is always altered through the influence of the not necessarily uniform acceleration. 


\section{$4 \quad$ Lamb shift for arbitrary stationary trajectories}

As mentioned in Sec. 2, it is possible with the same formalism to identify the contributions of vacuum fluctuations and radiation reaction to the Lamb shift of the two-level atom. It is interesting to ask the same questions for the energy shifts as for the relaxation rates: how are the two contributions $\left(\delta E_{a}\right)_{v f}$ and $\left(\delta E_{a}\right)_{r r}$ of (14) and (15) modified for an atom on an arbitrary stationary trajectory?

To investigate this problem we do not start directly from the formulas (14) and (15). Instead we use a relation between relaxation rates and energy shifts that was derived under very general assumptions in our previous paper [7]. Applied to the special model we are discussing here, it reads

$$
\begin{aligned}
& \left(\delta E_{a}\right)_{r r}=-\frac{1}{4 \pi} \sum_{b} \int_{-\infty}^{+\infty} d \omega^{\prime} \frac{1}{\omega^{\prime}} \Gamma_{a b}^{r r}\left(\omega^{\prime}\right)\left(\frac{\mathcal{P}}{\omega^{\prime}+\omega_{a b}}+\frac{\mathcal{P}}{\omega^{\prime}-\omega_{a b}}\right) . \\
& \left(\delta E_{a}\right)_{v f}=-\frac{1}{4 \pi} \sum_{b} \int_{-\infty}^{+\infty} d \omega^{\prime} \frac{1}{\omega^{\prime}} \Gamma_{a b}^{v f}\left(\omega^{\prime}\right)\left(\frac{\mathcal{P}}{\omega^{\prime}+\omega_{a b}}-\frac{\mathcal{P}}{\omega^{\prime}-\omega_{a b}}\right),
\end{aligned}
$$

where $\Gamma_{a b}^{v f}$ and $\Gamma_{a b}^{r r}$ have been defined in (24) and (17). With the help of this theorem, it is easy to formulate the analogue of Theorem 1 for the radiative energy shifts, especially for the relative shift $\Delta=\delta E_{+}-\delta E_{-}$of the two levels ("Lamb shift"), which is the measurable quantity:

Theorem 3: (a) The contribution of radiation reaction to the energy shift $\delta E_{a}$ is for all stationary trajectories equal to that of an atom at rest. (b) Radiation reaction does not contribute to the relative energy shift of a two-level atom:

$$
\Delta_{r r}=\left(\delta E_{+}\right)_{r r}-\left(\delta E_{-}\right)_{r r}=0
$$

Proof: Both conclusions follow simply from Eq. (30) together with (17).

Since equation (30) is not restricted to the two-level atom, part (a) of Theorem 3 can be extended to multi-level atoms. Part (b) will not remain true in the general case because of different coupling strengths of the energy levels to the field. However, there will be no influence of the motion to the relative shift and it will remain constant.

It is not so easy to obtain an analogue of Theorem 2, because of the divergence of the level shift of the atom at rest. We must first show that the additional level shift to the inertial one due to the influence of the motion is finite.

Theorem 4: The additional contribution $\left(\delta E_{a}\right)_{v f}-\left(\delta E_{a}\right)_{v f}^{\text {inert }}$ of vacuum fluctuations to $\delta E_{a}$ due to acceleration is finite for all stationary trajectories.

Proof: We want to show that

$$
\begin{aligned}
& \left|\left(\delta E_{a}\right)_{v f}-\left(\delta E_{a}\right)_{v f}^{\text {inert }}\right| \\
& =\left|-\frac{1}{4 \pi} \sum_{b} \int_{-\infty}^{+\infty} d \omega^{\prime}\left(\frac{\Gamma_{a b}^{v f}\left(\omega^{\prime}\right)}{\omega^{\prime}}-\frac{\Gamma_{a b}^{v f}\left(\omega^{\prime}\right)^{I}}{\omega^{\prime}}\right)\left(\frac{\mathcal{P}}{\omega^{\prime}+\omega_{a b}}-\frac{\mathcal{P}}{\omega^{\prime}-\omega_{a b}}\right)\right|<\infty
\end{aligned}
$$

We estimate the integrand for the case $\left|\omega^{\prime}\right| \rightarrow \infty$. Using equation (24) with (12) the integrand is proportional to

$$
\left|\frac{1}{\omega^{\prime 2}-\omega_{a b}^{2}} \int_{0}^{\infty} d u\left(\frac{1}{\sigma^{2}(u+i \epsilon)}+\frac{1}{\sigma^{2}(u-i \epsilon)}-\frac{1}{u^{2}+i \epsilon}-\frac{1}{u^{2}-i \epsilon}\right) \cos \left(\omega^{\prime} u\right)\right|
$$

with the abbreviation $\sigma^{2}(u+i \epsilon):=(\Delta t+i \epsilon)^{2}-|\Delta \vec{x}|^{2}$. Because of the stationary motion the right side depends only on the proper time interval $u=\tau-\tau^{\prime}\left(\sigma^{2}(u)=u^{2}\right.$ for an inertial atom). With the substitution $x=\omega^{\prime} u$ we can expand

$$
\frac{1}{\sigma^{2}(x)}=\frac{\omega^{\prime 2}}{x^{2}\left(1+A \frac{x^{2}}{\omega^{\prime 2}}+\mathcal{O}\left(\frac{x^{4}}{\omega^{\prime 4}}\right)\right)} \approx \frac{\omega^{\prime 2}}{x^{2}}\left(1-A \frac{x^{2}}{\omega^{\prime 2}}+\mathcal{O}\left(\frac{x^{4}}{\omega^{\prime 4}}\right)\right)
$$

in the limit $\left|\omega^{\prime}\right| \rightarrow \infty$. A short calculation shows that the integrand of (33) goes faster to zero as $\frac{1}{\omega^{\prime 2}}$ and hence the total integral converges. 
This fact agrees with our expectation that the additional energy shift due to an accelerated motion should be a continuous function of the acceleration without divergences, so that for weak acceleration this additional contribution goes continuously to zero.

Theorem 5: The contribution of vacuum fluctuations to $\delta E_{a}$ differs for any accelerated stationary trajectory from that of an atom at rest.

Proof: Using equation (14) with the linear susceptibility of the atom (11) we can write for the additional contribution to the inertial value of the energy shift

$$
\left(\delta E_{a}\right)_{v f}-\left(\delta E_{a}\right)_{v f}^{\text {inert }}=\mu^{2}\left|\left\langle a\left|R_{2}^{f}(0)\right| b\right\rangle\right|^{2} \int_{0}^{\infty} d u\left[C^{F}(u)-C_{\text {inert }}^{F}(u)\right] \sin \omega_{a b} u .
$$

The integral appearing is just the Fourier sine transform of $C^{F}(u)-C_{\text {inert }}^{F}(u)$. In Theorem 4 we have shown that the integral converges, so that the Fourier transform exists and is unique. Equation (28) ensures that there will always be a contribution of vacuum fluctuations to the energy shift of an atom at rest for any accelerated stationary motion.

It is easy to see, that for the two level system the relative energy shift is given by

$$
\Delta_{v f}=\left(\delta E_{+}\right)_{v f}-\left(\delta E_{-}\right)_{v f}=2\left(\delta E_{+}\right)_{v f} .
$$

This agrees with the total Lamb shift $\Delta$ for a two-level atom, since according to (32), radiation reaction does not contribute to the relative shift of the two levels. Because of the divergence of the Lamb shift, it is useful to split the Lamb shift in the diverging part $\Delta_{\text {inert }}$, which is equal to the Lamb shift of an atom at rest, and the non-diverging rest $\mathcal{D}$ appearing for any accelerated moving atom

$$
\Delta=\Delta_{\text {inert }}+\mathcal{D} \text {. }
$$

Below we will evaluate $\mathcal{D}$ for a special case.

Result: The Lamb shift $\Delta$ of an accelerated two-level atom always deviates from its inertial value, although only the contribution of vacuum fluctuations gives rise to this modification.

\section{Application: Atom in circular motion}

To illustrate the use of our theorems, we consider the concrete situation of an atom in circular motion on the trajectory

$$
\begin{gathered}
t(\tau)=\gamma \tau, \quad x(\tau)=R \cos (\omega \gamma \tau), \\
y(\tau)=R \sin (\omega \gamma \tau), \quad z(\tau)=0
\end{gathered}
$$

with constant radius $R$ and magnitude of velocity $v$. In (39), $\gamma=\left(1-v^{2}\right)^{-1 / 2}$ is the usual relativistic factor, $\omega=v / R$ is the angular velocity, and $a=v^{2} \gamma^{2} / R=\omega^{2} \gamma^{2} R$ the magnitude of acceleration. The "circular Unruh effect" for a two-level system on the trajectory (39) has been discussed by several authors [5, 9, 10]. We will discuss separately the contributions of vacuum fluctuations and radiation reaction to the Unruh effect and spontaneous emission. Furthermore, we will be able to study the evolution of the atomic population to its equilibrium value and identify the Einstein coefficients for the spontaneous processes. Finally, application of Eqs. (31) and (30) will allow us to determine the Lamb shift of an atom in circular motion.

The statistical functions of the field can be evaluated with the help of

$$
\left|\mathbf{x}(\tau)-\mathbf{x}\left(\tau^{\prime}\right)\right|=2 R\left|\sin \left(\omega \gamma \frac{\tau-\tau^{\prime}}{2}\right)\right| .
$$

We find

$$
\begin{aligned}
& \chi^{F}\left(\mathbf{x}(\tau), \mathbf{x}\left(\tau^{\prime}\right)\right)=-\frac{i}{8 \pi} \frac{\omega \gamma}{\left|\sin \left(\omega \gamma \frac{\tau-\tau^{\prime}}{2}\right)\right|} \delta\left(\tau-\tau^{\prime}\right) \\
& C^{F}\left(\mathbf{x}(\tau), \mathbf{x}\left(\tau^{\prime}\right)\right)=-\frac{1}{8 \pi^{2}}\left(\frac{1}{\gamma^{2}\left(\tau-\tau^{\prime}\right)^{2}-4 R^{2} \sin ^{2}\left(\omega \gamma \frac{\tau-\tau^{\prime}}{2}\right)+i \epsilon}\right. \\
& \left.+\frac{1}{\gamma^{2}\left(\tau-\tau^{\prime}\right)^{2}-4 R^{2} \sin ^{2}\left(\omega \gamma \frac{\tau-\tau^{\prime}}{2}\right)-i \epsilon}\right)
\end{aligned}
$$


It is easy to verify by explicit calculation that the contribution of radiation reaction to $\left\langle d H_{A} / d \tau\right\rangle$ is given indeed by (17), as stated by Theorem 1 .

The contribution of vacuum fluctuations is more difficult to obtain. It is calculated from Eq. (何) via the residue theorem so that we need to know the zeroes of the denominator. This leads to an equation of the form $x^{2}=v^{2} \sin ^{2} x$, which is not analytically solvable. The problem becomes tractable in the "high velocity limit" [9, 10]. For $v \gtrsim 0.85$, we can expand the sine to find the zero with the smallest imaginary part (besides $x=0$ ). Because the exponential in the numerator of the resulting integral, zeroes with larger imaginary part can be neglected. In this way, we obtain

$$
\begin{aligned}
\left\langle\frac{d H(\tau)}{d \tau}\right\rangle_{v f}=-\frac{\mu^{2}}{2 \pi} & \sum_{\omega_{a}>\omega_{b}}\left(\frac{\omega_{a b}^{2}}{2}+\frac{a \omega_{a b}}{4 \sqrt{3}} A e^{-2 \sqrt{3} B \frac{\omega_{a b}}{a}}\right)\left|\left\langle a\left|R_{2}^{f}(0)\right| b\right\rangle\right|^{2} \\
& -\frac{\mu^{2}}{2 \pi} \sum_{\omega_{a}<\omega_{b}}\left(\frac{\omega_{a b}^{2}}{2}+\frac{a\left|\omega_{a b}\right|}{4 \sqrt{3}} A e^{-2 \sqrt{3} B \frac{\left|\omega_{a b}\right|}{a}}\right)\left|\left\langle a\left|R_{2}^{f}(0)\right| b\right\rangle\right|^{2}
\end{aligned}
$$

where

$$
A=1+\frac{3}{5}(v \gamma)^{-2}, \quad B=1-\frac{1}{5}(v \gamma)^{-2} .
$$

The total rate of change of the atomic excitation energy can be found by adding the contributions of vacuum fluctuations and radiation reaction:

$$
\begin{aligned}
\left\langle\frac{d H(\tau)}{d \tau}\right\rangle_{t o t}=-\frac{\mu^{2}}{2 \pi} & \sum_{\omega_{a}>\omega_{b}}\left(\omega_{a b}^{2}+\frac{a \omega_{a b}}{4 \sqrt{3}} A e^{-2 \sqrt{3} B \frac{\omega_{a b}}{a}}\right)\left|\left\langle a\left|R_{2}^{f}(0)\right| b\right\rangle\right|^{2} \\
& -\frac{\mu^{2}}{2 \pi} \sum_{\omega_{a}<\omega_{b}} \frac{a\left|\omega_{a b}\right|}{4 \sqrt{3}} A e^{2 \sqrt{3} B \frac{\left|\omega_{a b}\right|}{a}}\left|\left\langle a\left|R_{2}^{f}(0)\right| b\right\rangle\right|^{2}
\end{aligned}
$$

We recognize that spontaneous excitation $\left(\omega_{a}<\omega_{b}\right)$ is possible as well as spontaneous de-excitation $\left(\omega_{a}>\omega_{b}\right)$. As stated by the contents of theorems 1 and 2 , the balance between vacuum fluctuations and radiation reaction which is present for an inertial atom becomes upset.

From Eq. (45), it is possible to get in second order in $\mu$ a differential equation for $\left\langle H_{A}\right\rangle$, (cf. [2])

$$
\left\langle\frac{d H(\tau)}{d \tau}\right\rangle=-\frac{\mu^{2}}{8 \pi} \omega_{0}\left\{\frac{\omega_{0}}{2}+\left(1+\frac{A}{2 \sqrt{3}} \frac{a}{\omega_{0}} e^{-2 \sqrt{3} B \frac{\omega_{0}}{a}}\right)\langle H(\tau)\rangle\right\}
$$

The solution

$$
\begin{aligned}
\langle H(\tau)\rangle=- & \frac{\omega_{0}}{2}+\frac{\omega_{0}}{2}\left[1+\frac{2 \sqrt{3}}{A} \frac{\omega_{0}}{a} e^{2 \sqrt{3} B \frac{\omega_{0}}{a}}\right]^{-1} \\
& +\left(\langle H(0)\rangle+\frac{\omega_{0}}{2}-\frac{\omega_{0}}{2}\left[1+\frac{2 \sqrt{3}}{A} \frac{\omega_{0}}{a} e^{2 \sqrt{3} B \frac{\omega_{0}}{a}}\right]^{-1}\right) e^{-\Gamma \tau}
\end{aligned}
$$

gives the time evolution of the mean atomic excitation energy. The decay rate is

$$
\Gamma=\frac{\mu^{2}}{8 \pi} \omega_{0}\left(1+\frac{A}{2 \sqrt{3}} \frac{a}{\omega_{0}} e^{-2 \sqrt{3} B \frac{\omega_{0}}{a}}\right)
$$

The second term in the bracket represents the modification of the inertial decay constant

$$
\Gamma_{\text {inert }}=\frac{\mu^{2}}{8 \pi} \omega_{0}
$$

From (47) we see that the system evolves towards an equilibrium population

$$
\left\langle H_{A}\right\rangle=-\frac{1}{2} \omega_{0}+\frac{\omega_{0}}{2}\left(1+\frac{2 \sqrt{3}}{A} \frac{\omega_{0}}{a} e^{2 \sqrt{3} B \frac{\omega_{0}}{a}}\right)^{-1},
$$


representing a mean excitation above the ground state $-\frac{1}{2} \omega_{0}$.

Comparison of (47) with the solution of an appropriately defined rate equation (cf. Eq. (65) in Ref. [2]) allows the identification of the two Einstein coefficients $A_{\downarrow}$ and $A_{\uparrow}$ for spontaneous emission and the Unruh effect. We obtain

$$
\begin{gathered}
A_{\downarrow}=\frac{\mu^{2}}{8 \pi} \omega_{0}\left[1+\frac{A}{4 \sqrt{3}} \frac{a}{\omega_{0}} e^{-2 \sqrt{3} B \frac{\omega_{0}}{a}}\right], \\
A_{\uparrow}=\frac{\mu^{2}}{8 \pi} a \frac{A}{4 \sqrt{3}} e^{-2 \sqrt{3} B \frac{\omega_{0}}{a}} .
\end{gathered}
$$

The spontaneous emission rate $A_{\downarrow}$ shows a modification of the value $\mu^{2} \omega_{0} / 8 \pi$ for an atom at rest, in accordance with Theorem 2 . Spontaneous excitation (the "circular Unruh effect") occurs with a rate $A_{\uparrow}$. Its value is in accordance with previous results [9, 10],

Following [9] we can define an effective temperature by

$$
k T_{\text {eff }}=\omega_{0}\left[-\ln \left(A_{\uparrow} / A_{\downarrow}\right)\right]^{-1}
$$

For $\omega_{0} \gg a$ the equilibrium population of the upper level relative to the lower level is then

$$
\frac{A_{\uparrow}}{A_{\downarrow}} \simeq \frac{1}{4 \sqrt{3}} \frac{a}{\omega_{0}} e^{-2 \sqrt{3} \frac{\omega_{0}}{a}}
$$

leading to a temperature

$$
k T_{e f f} \simeq \frac{a}{2 \sqrt{3}}
$$

which is higher by a factor $\frac{1}{\sqrt{3}} \pi$ than the Unruh temperature for linear acceleration $T_{U}=\frac{a}{2 \pi}$.

With the help of Eqs. (31) and (30), we can determine from $\left\langle d H_{A} / d \tau\right\rangle_{v f}$ and $\left\langle d H_{A} / d \tau\right\rangle_{r r}$ the contributions of vacuum fluctuations and radiation reaction to the Lamb shift of the circularly moving two-level atom. We know already that the contribution of radiation reaction is the same as for an inertial atom and thus does not contribute to the relative energy shift:

$$
\Delta_{r r} \equiv\left(\delta E_{+}\right)_{r r}-\left(\delta E_{-}\right)_{r r}=0 .
$$

The contribution of vacuum fluctuations can be calculated with (31) and (43):

$$
\left(\delta E_{a}\right)_{v f}=\frac{\mu^{2}}{8 \pi^{2}} \sum_{b}\left|\left\langle a\left|R_{2}^{f}(0)\right| b\right\rangle\right|^{2} \int_{0}^{+\infty} d \omega^{\prime}\left(\omega^{\prime}+\frac{a}{2 \sqrt{3}} A e^{-2 \sqrt{3} B \frac{\omega^{\prime}}{a}}\right)\left(\frac{\mathcal{P}}{\omega^{\prime}+\omega_{a b}}-\frac{\mathcal{P}}{\omega^{\prime}-\omega_{a b}}\right) .
$$

The Lamb shift $\Delta$, i. e. the relative shift of the two levels can be found by evaluating the $b$ summation in (57) for each of the levels separately:

$$
\begin{aligned}
\Delta & =\Delta_{v f} \equiv\left(\delta E_{+}\right)_{v f}-\left(\delta E_{-}\right)_{v f} \\
& =\frac{\mu^{2}}{16 \pi^{2}} \int_{0}^{+\infty} d \omega^{\prime}\left[\omega^{\prime}+\frac{a}{2 \sqrt{3}} A e^{-2 \sqrt{3} B \frac{\omega^{\prime}}{a}}\right]\left(\frac{\mathcal{P}}{\omega^{\prime}+\omega_{0}}-\frac{\mathcal{P}}{\omega^{\prime}-\omega_{0}}\right) .
\end{aligned}
$$

The first one of the two terms appearing in square brackets, coincides with the Lamb shift $\Delta_{\text {inert }}$ for an atom at rest. The second one represents the influence of the circular acceleration, which gives the finite correction $\mathcal{D}$ to the level shift. With the help of Eq. (3.354.3) from Ref. [14], it can be evaluated in closed form. We obtain

$$
\mathcal{D}=\frac{a \mu^{2}}{64 \sqrt{3} \pi^{2}}\left(e^{-2 \sqrt{3} B \omega_{0} / a} \overline{\operatorname{Ei}}\left(2 \sqrt{3} B \omega_{0} / a\right)-e^{2 \sqrt{3} B \omega_{0} / a} \overline{\operatorname{Ei}}\left(-2 \sqrt{3} B \omega_{0} / a\right)\right) .
$$

$\overline{\text { Ei }}$ denotes the principal value of the exponential integral function 14. A plot of the acceleration-induced correction $\mathcal{D}$ to the inertial emission rate $\Gamma_{\text {inert }}=\frac{\mu^{2}}{8 \pi} \omega_{0}$ as a function of $\frac{a}{\omega_{0}}$ is shown in Fig. 1.

For an estimation of the order of magnitude we consider an electron, moving in a circular orbit perpendicular to a uniform magnetic field $\left|\mathbf{B}_{0}\right|=B_{0}$ (cf. [9]). The magnetic and electric fields in the co-moving frame are

$$
\mathbf{B}_{0}^{\prime}=\gamma \mathbf{B}_{0}
$$




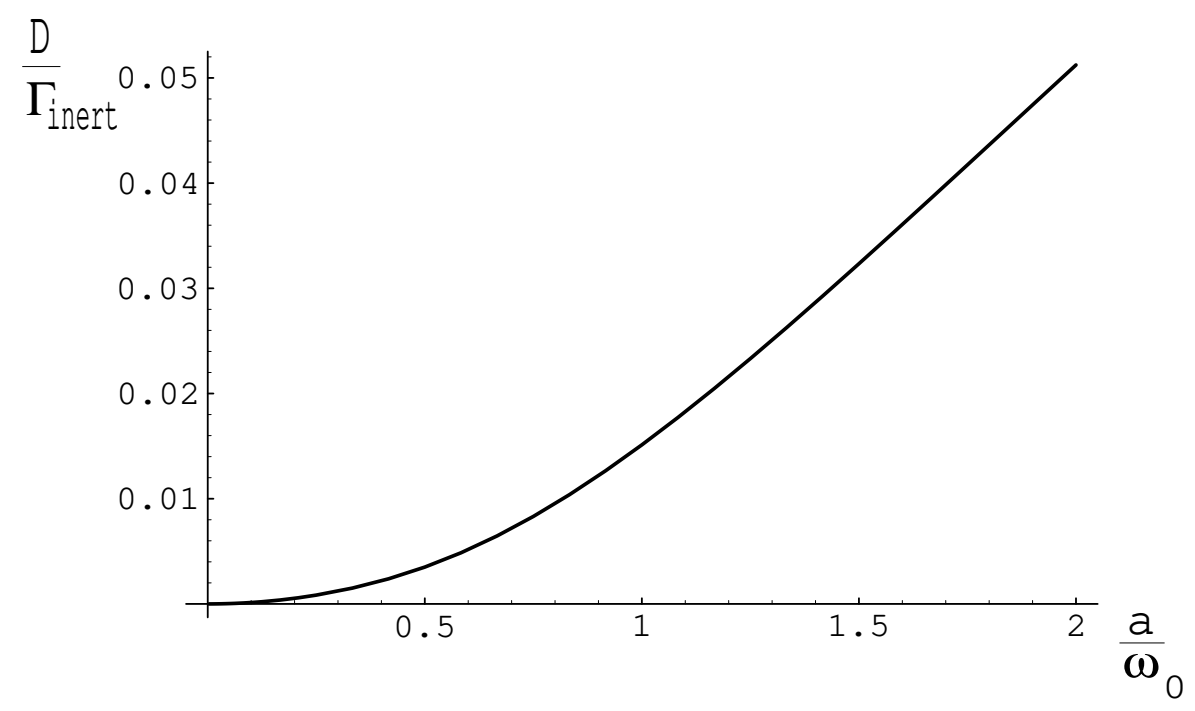

Figure 1: The acceleration-induced energy shift $\mathcal{D}$ of a two-level atom in circular motion $\left(\Gamma_{\text {inert }}=\frac{\mu^{2}}{8 \pi} \omega_{0}\right)$.

$$
\mathbf{E}_{0}^{\prime}=\gamma \mathbf{v} \times \mathbf{B}_{0}
$$

The splitting of the two energy levels "spin up" and "spin down" in the rest frame is given by

$$
\omega_{0}=\frac{e}{2 m}|g| B_{0}^{\prime} .
$$

The proper acceleration of an electron moving with velocity $\mathrm{v}$ is

$$
a=\frac{e}{m} E_{0}^{\prime}=v \frac{e}{m} B_{0}^{\prime}
$$

Therefore we get for ultra-relativistic electrons $(v \simeq 1, \mathrm{~g}=2)$ the ratio $a / \omega_{0} \approx 1$, which gives rise to a correction $\mathcal{D}$ of the relative energy shift with value $\mathcal{D} / \Gamma_{\text {inert }} \approx 1.5 \%$.

\section{References}

[1] W. G. Unruh, Phys. Rev. D 14, 870 (1976).

[2] J. Audretsch and R. Müller, Phys. Rev. A 50, 1755, (1994).

[3] J. Dalibard, J. Dupont-Roc, and C. Cohen-Tannoudji, J. Physique 43, 1617 (1982).

[4] D. W. Sciama, P. Candelas, and D. Deutsch, Adv. Phys. 30, 327 (1981).

[5] J. R. Letaw, Phys. Rev. D23, 1709 (1981).

[6] J. Audretsch and R. Müller, Phys. Rev. A. 52, 629 (1995).

[7] J. Audretsch, R. Müller and M. Holzmann, Phys. Lett. A 199, 151 (1995).

[8] J. Letaw and J. Pfautsch, Phys. Rev. D 22, 1345 (1980).

[9] J. S. Bell and J. M. Leinaas, Nucl. Phys. B 212, 131 (1983).

[10] S. Takagi, Prog. Theor. Phys. Suppl. 88, 1 (1986).

[11] R. H. Dicke, Phys. Rev. 93, 99 (1954).

[12] L. Allen and J. H. Eberly, Optical resonance and two-level atoms Dover, New York (1975). 
[13] J. Dalibard, J. Dupont-Roc, and C. Cohen-Tannoudji, J. Physique 45, 637 (1984).

[14] I. S. Gradshteyn and I. M. Ryzhik, Table of Integrals, Series, and Products, Academic Press, Orlando (1980). 